\title{
Reproducibility of glycaemic thresholds for activation of counterregulatory hormones and hypoglycaemic symptoms in healthy subjects
}

\author{
H. Vea ${ }^{1}$, R. Jorde ${ }^{3}$, G.Sager ${ }^{2}$, S. Vaaler ${ }^{5}$ and J.Sundsfjord ${ }^{4}$ \\ ${ }^{1}$ Institute of Clinical Medicine, ${ }^{2}$ Institute of Medical Biology, University of Troms $\emptyset,{ }^{3}$ Department of Medicine \\ ${ }^{4}$ Department of Clinical Chemistry, University Hospital of Troms $\varnothing$, Troms $\varnothing$ \\ ${ }^{5}$ Hormone Laboratory, A ker University Hospital, Oslo, Norway
}

\begin{abstract}
Summary. Nine healthy subjects were studied on two separate occasions, at least two weeks apart, using the glucose clamp technique to produce a gradual hypoglycaemia. Glucose thresholds for neuroendocrine and symptom responses varied up to $1.5 \mathrm{mmol} / \mathrm{l}$ between subjects. There was a significant correlation between individual glucose thresholds on day 1 and 2 for adrenaline $(p=0.0008)$, growth hormone $(p=0.007)$ and pancreatic polypeptide $(p=0.02)$, and for autonomic $(p=0.018)$ and neuroglycopoenic $(p=0.023)$ symptoms, whereas no significant correlations were found
\end{abstract}

for glucagon and cortisol. The mean intra-individual differences in glucose thresholds between day 1 and 2 were 0.22 $\mathrm{mmol} / \mathrm{l}$ for the hormones and $0.25 \mathrm{mmol} / \mathrm{l}$ for the symptoms. We conclude that healthy subjects differ in hypoglycaemic thresholds, and that the difference reflects individual variation.

Key words: Autonomic symptoms, counterregulatory hormones, glucose thresholds, hypoglycaemia, neuroglycopoenia.
Regulation of glycaemic thresholds for activation of counterregulatory hormones and symptoms is not well understood, but it appears that daily glucose levels are of importance. Accordingly, Amiel et al. [1] reported that a period of intensive insulin therapy significantly increased the glycaemic thresholds (i.e. lower blood glucose was needed to elicit the hypoglycaemic responses) in a group of young Type 1 (insulin-dependent) diabetic patients, and Mokan et al. [2] found the thresholds to be at a plasma glucose level approximately $1 \mathrm{mmol} / 1$ higher in diabetic patients with poor compared to those with good glycaemic control. Similarly, in a case report on a patient with an insulinoma [3], the glucose threshold for adrenaline changed from 2.6 to $3.7 \mathrm{mmol} / 1$ when the blood glucose level was normalized following removal of the tumour.

However, average daily blood glucose levels cannot be the only factor regulating the threshold for hypoglycaemic responses. In a group of patients with functional hypoglycaemia, normal $\mathrm{HbA}_{1 \mathrm{c}}$ and normal blood glucose levels on self-testing, the hypoglycaemic threshold was found to be lower than in control subjects in a study by Snorgaard et al. [4].

When testing whether the rate of glucose fall affected counterregulation, Amiel et al. [5] found that healthy subjects exhibited a wide range in glucose thresholds for adrenaline release. Whether this apparent variation in hypoglycaemic thresholds between healthy subjects truly reflects individual differences, or is simply the result of inaccurate methods, is not known.

The purpose of this study was, therefore, to look at the reproducibility of hypoglycaemic thresholds for activation of neuroendocrine responses and symptoms in healthy subjects by comparing two consecutive studies using the glucose clamp technique to produce a gradual decline in blood glucose levels.

\section{Subjects and methods}

Nine healthy subjects were studied on two occasions. Both studies were performed identically, at least 2 weeks apart. Data on age, sex, BMI, smoking, and fasting blood glucose levels are given in Table 1. None of the subjects were taking any medication. The subjects were admitted after an overnight fast to the metabolic research laboratory. A cannula for constant infusion of rapidacting insulin $\left(1.5 \mathrm{mU} \cdot \mathrm{kg}^{-1} \cdot \mathrm{min}^{-1}\right)$ and $24 \%$ glucose was placed in the antecubital vein of the left arm. A second cannula for blood sampling was inserted into a dorsal hand vein on the right arm. This arm was placed in a heated box $\left(63^{\circ} \mathrm{C}\right)$ to arterialize the venous blood and the intravenous line was kept patent with $0.9 \% \mathrm{NaCl}(0.5 \mathrm{ml} / \mathrm{min})$.

After a resting and stabilizing period of approximately $30 \mathrm{~min}$, the tests were started between 09.00 and 10.30 hours. Initially the plasma glucose level was stabilized between 4.5 and $5.5 \mathrm{mmol} / \mathrm{h}$ for $30 \mathrm{~min}$ and subsequently allowed to fall gradually during $240 \mathrm{~min}$ to $2.0 \mathrm{mmol} / \mathrm{l}$ (giving a rate of decline of $0.5 \mathrm{mmol} / \mathrm{l}$ per $40 \mathrm{~min}$ ). All subjects were informed that the blood glucose level would be lowered, without knowing the level at any stage. If serious clinical symptoms of hypoglycaemia (falling asleep, diplopia, slurred and in- 
Table 1. Clinical characteristics of the participants

\begin{tabular}{llllll}
\hline Subject & $\begin{array}{l}\text { Age } \\
\text { (years) }\end{array}$ & Sex & $\begin{array}{l}\text { BMI } \\
\left(\mathrm{kg} / \mathrm{m}^{2}\right)\end{array}$ & Smoking & $\begin{array}{l}\text { Fasting blood glucose } \\
\text { Study day 1/day 2 } \\
\text { (mmol/l) }\end{array}$ \\
\hline 1 & 26 & M & 20.5 & no & $5.4 / 4.9$ \\
2 & 20 & M & 20.7 & no & $4.6 / 5.4$ \\
3 & 23 & F & 23.4 & no & $5.2 / 5.5$ \\
4 & 23 & M & 19.4 & no & $5.3 / 5.2$ \\
5 & 27 & M & 21.2 & no & $5.5 / 5.5$ \\
6 & 29 & M & 26.0 & no & $5.5 / 5.1$ \\
7 & 25 & F & 21.5 & no & $5.5 / 4.9$ \\
8 & 27 & F & 19.1 & yes & $4.7 / 4.7$ \\
9 & 27 & F & 20.3 & no & $4.5 / 4.8$ \\
\hline
\end{tabular}

coordinate speech etc.) occurred before the plasma glucose had fallen to $2.0 \mathrm{mmol} / \mathrm{l}$, the insulin infusion was stopped and the blood glucose normalized by glucose infusion. Plasma glucose was measured repeatedly, and the desired glucose level achieved by adjusting the glucose infusion rate accordingly.

Blood samples were drawn and a symptorn questionnaire completed at 5-min intervals during the first $30 \mathrm{~min}$ and thereafter every $10 \mathrm{~min}$.

Written informed consent was obtained from each subject before participation. The protocol was approved by the regional ethical committee.

\section{Clinicalmeasurements}

Plasma glucose was measured at the bedside by the glucose oxidase method on a Beckman glucose analyser (Fullerton, Calif., USA). Blood for plasma adrenaline was collected in tubes containing glutathione, centrifuged at $4^{\circ} \mathrm{C}$, frozen at $-70^{\circ} \mathrm{C}$, and later analysed by HPLC [6], with a detection limit of $5.5 \mathrm{pmol} / \mathrm{l}$. Serum growth hormone was analysed by immuno-radiometric assay (IRMA) developed by the Hormone Laboratory, Aker University Hospital, Oslo, Norway. This assay has a coefficient of variation (CV) of $10.0 \%$ [7]. Determination of serum cortisol was based on enhanced luminescence (Amerlite Diagnostics Ltd, Amersham, Buckinghamshire, UK), with a CV of $4.3 \%$. Blood for determination of plasma glucagon was collected in ice-chilled tubes containing $10 \%$ trasylol/EDTA, centrifuged at $4{ }^{\circ} \mathrm{C}$, frozen, and later analysed by RIA $(\mathrm{CV}, 12.0 \%)[8]$. Serum pancreatic polypeptide $(\mathrm{PP})$ was frozen and later measured by RIA (CV, $12.0 \%)[9]$.

\section{Symptoms}

Prior to the study the subjects were given instructions for completing the visual scale analogue symptom questionnaire. Eight symptoms (dizziness, weakness, tiredness, difficulty concentrating, trembling, sweating, pounding heart, anxiety) were scored on a line from 0 (no symptoms) to a maximum of $15 \mathrm{~cm}$. Consistent with the categorization recommended by Hepburn et al. [10] the first four symptoms were considered neuroglycopoenic and the other four were considered autonomic. The order of the symptoms was systematically changed on each questionnaire. The symptoms in each of the two groups were added together to give an autonomic and a neuroglycopoenic score.

\section{Statistical analysis}

Glucose thresholds for each hormone or symptom are given as the mean between the plasma glucose level where responses were more than 2 SD above baseline, followed by an unequivocal, sustained increase, and the preceding plasma glucose. In a few cases the preceding plasma glucose level was lower than the mean between the two, and in these cases regarded as the threshold value. Some patients had an initial decrease or spike in the first hormone samples in which case the values which followed were chosen as baseline.
Correlations between the glucose thresholds day 1 and day 2 were performed by ordinary least squares regression. Thresholds are given as mean \pm SEM

Differences in glucose thresholds for hormones and symptoms were evaluated with two-tailed Wilcoxon's rank sum tests and $p<0.05$ was considered statistically significant.

\section{Results}

A comparable and gradual decrease in plasma glucose was obtained on both study days in all nine subjects (Fig.1).

Except for growth hormone in three subjects and PP and cortisol in a fourth subject, glucose thresholds for all hormones could be assigned on both days. Similarly, glucose thresholds for autonomic symptoms were found in eight, and thresholds for neuroglycopoenic symptoms in seven of the nine subjects (Fig. 2).

There was a significant correlation between glucose thresholds day 1 and 2 for adrenaline $(r=0.91$, $p=0.0008)$, growth hormone $(r=0.93, p=0.007)$ and $\mathrm{PP}(r=0.79, \quad p=0.02)$, and for autonomic $(r=0.80$, $p=0.018)$ and neuroglycopoenic symptoms $(r=0.82$, $p=0.023)$. There was a non-significant correlation for glu$\operatorname{cagon}(r=0.62, p=0.074)$ and cortisol $(r=0.40, p=0.33)$. The mean difference between the glycaemic thresholds day 1 and 2 was $0.22 \pm 0.03 \mathrm{mmol} / 1$ for the hormones and $0.25 \pm 0.05 \mathrm{mmol} / \mathrm{l}$ for the symptoms.

Dividing the group by sex still demonstrated a positive correlation between thresholds day 1 and 2 for all hormones and symptoms.

No correlations were seen between initial plasma glucose levels and glucose thresholds, or between body mass index and glucose thresholds.

The thresholds for the hormones differed, being $3.8 \pm 0.11 \mathrm{mmol} / \mathrm{l}$ for adrenaline, $3.6 \pm 0.08 \mathrm{mmol} / 1$ for cortisol, $3.7 \pm 0.12 \mathrm{mmol} / \mathrm{l}$ for growth hormone, $3.7 \pm 0.10 \mathrm{mmol} / \mathrm{l}$ for glucagon and $3.5 \pm 0.10 \mathrm{mmol} / \mathrm{l}$ for PP (the two days combined). The mean cortisol and PP thresholds on both days were at a significantly $(p<0.02)$ lower plasma glucose level than the adrenaline threshold.

The mean threshold for neuroglycopoenic symptoms $(3.4 \pm 0.14 \mathrm{mmol} / \mathrm{l})$ was not at a significantly lower plasma glucose level than that for the autonomic symptoms $(3.6 \pm 0.11 \mathrm{mmol} / \mathrm{l})$.

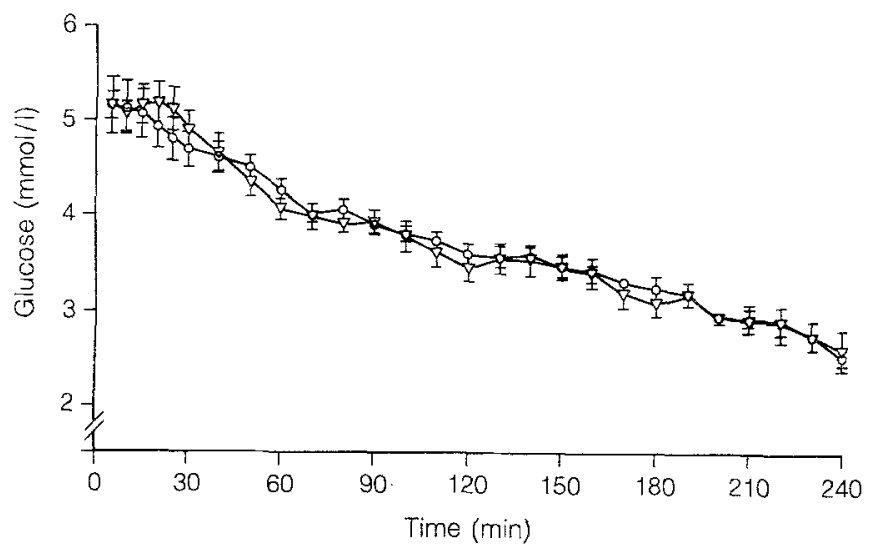

Fig.1. Mean arterialized venous plasma glucose concentrations $( \pm$ SEM) during the hyperinsulinaemic clamp in nine diabetic patients on day $1(0-0)$ and day $2(\nabla-\nabla)$ 

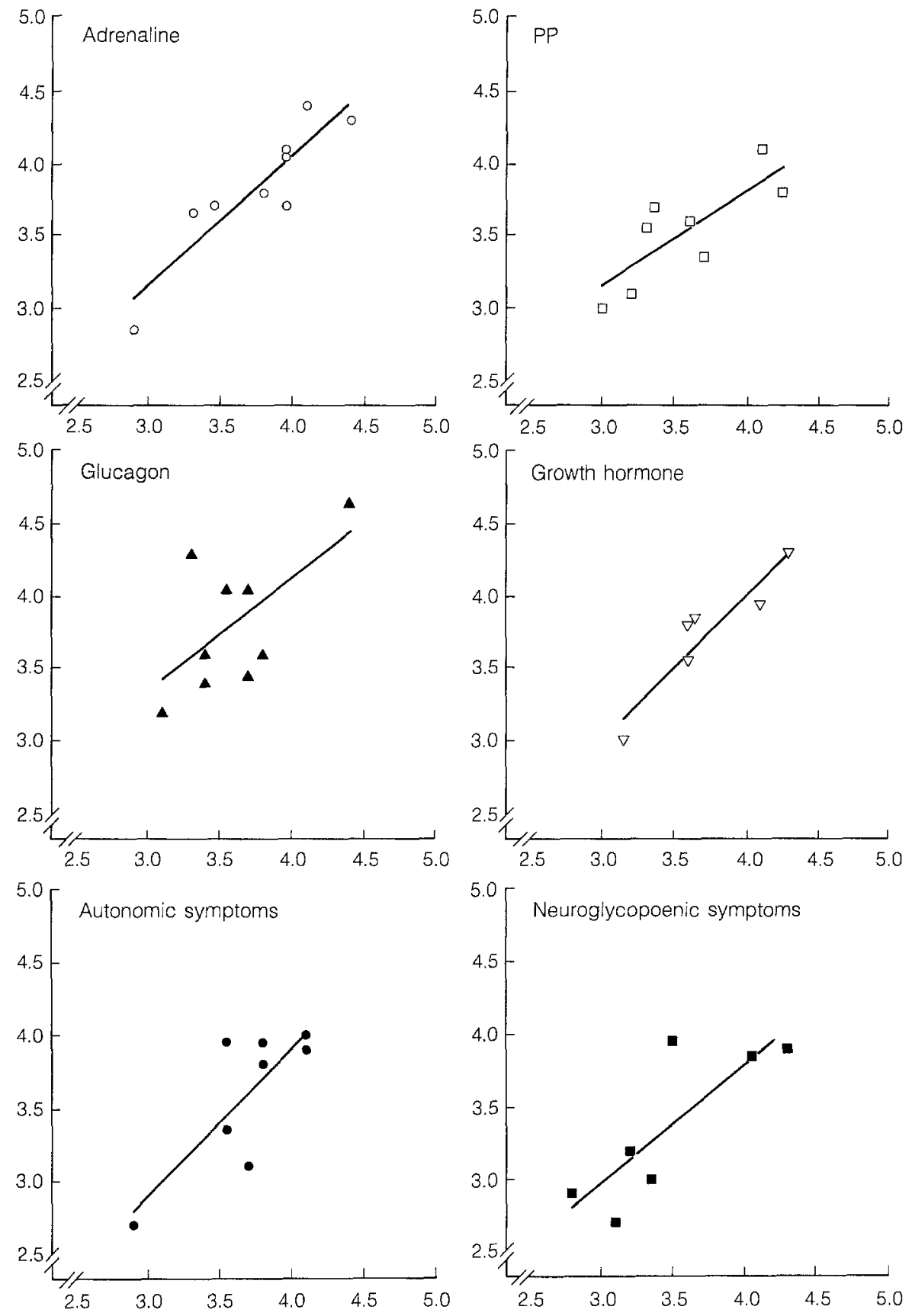

Fig. 2. Correlation between hypoglycaemic thresholds (plasma glucose level in $\mathrm{mmol} / \mathrm{l}$ ) day 1 (along the abscissa) and day 2 (along the coordinate) for adrenaline ( $r=0.91$, $p=0.0008)$, pancreatic polypeptide (PP) $(r=0.79, p=0.02)$, growth hormone $(r=0.93, p=0.007)$, glucagon $(r=0.62$, $p=0.074)$, autonomic symptoms $(r=0.80$, $p=0.018)$ and neuroglycopoenic symptoms $(r=0.82, p=0.023)$. Cortisol $(r=0.40$, $p=0.33$ ) is not illustrated

\section{Discussion}

To our knowledge this is the first study examining the reproducibility of the glucose clamp technique to determine glucose thresholds for hypoglycaemic responses.

The method used, a constant insulin infusion and adjusted glucose infusion after blood glucose measurements every 5 to $10 \mathrm{~min}$, should ideally allow plasma glucose to fall gradually, as does a straight line. Of course, this is not always the case, and is highly dependent on the expertise of the investigator monitoring the clamp. Usually a decline in plasma glucose of approximately $0.1 \mathrm{mmol} / \mathrm{l}$ per $10 \mathrm{~min}$ is obtained, but occasional levelling off or sharp drops do occur. In our hands the method gave reproducible results with a mean difference in glycaemic thresholds between the two separate tests of $0.2 \mathrm{mmol} / \mathrm{l}$. The problems associ- ated with trying to achieve an ideal blood glucose fall is the most likely cause for this intra-individual difference.

The possible influence of hormone assay variation appears to be minor, since at the threshold point the increase in hormone levels usually far exceeded the baseline plus 2 SD. Thus, the modified glucose clamp technique is a suitable tool for further studies on factors affecting the hypoglycaemic thresholds.

Furthermore, healthy individuals do differ in the glycaemic thresholds both for counterregulatory hormone release and recognition of hypoglycaemic symptoms. These differences should not be ascribed to method inaccuracy.

The reason for this individual variation is difficult to explain. Firstly, individual differences as the result of study group heterogenicity should be ruled out. After our study was completed, two abstracts have been published showing that males have hypoglycaemic thresholds at 
higher glucose levels than females (regardless of menstrual phase) [11,12]. The same tendency towards thresholds at lower glucose levels in females was also seen in our study, and thus may have influenced the level of correlation between the two days. However, a possible sex difference does not alter our conclusion, since a positive correlation between thresholds on day 1 and 2 was found for all the hormones and symptoms even after dividing the group into males and females.

Secondly, differences in daily blood glucose levels could, as in diabetic patients [1,2], possibly result in different glycaemic thresholds. Although fasting blood glucose levels showed no correlation with the glycaemic thresholds, multiple blood glucose determinations for several days would be needed to rule out this possibility.

Our slow fall clamp technique results in glucose thresholds where the effect of time to elicit a response has not been taken into account. Thus, if the blood glucose had been clamped for some time at a level above the "threshold", a significant response might have occurred. Furthermore, the slow glucose fall may possibly cause cerebral adaptation to the hypoglycaemia giving higher thresholds that what might have been found with a more rapid blood glucose fall. However, the thresholds found in our study are similar, or even occur at somewhat higher plasma glucose levels than those reported by others $[13,14]$, and the above-mentioned factors are probably not of major importance when determining the glucose thresholds.

The insulin concentration may affect the symptoms and hormonal responses to hypoglycaemia as shown by Kerr et al. [15]. In their study the two insulin doses used to show this effect differed three-fold and did not affect the adrenaline response. Unfortunately, insulin levels were not measured in our study. The insulin dose was given according to weight and therefore should result in fairly similar circulating levels. Although we cannot rule out an effect of different insulin levels on the inter-individual threshold differences, we believe this effect to have been minor, and for adrenaline non-existant.

The clamp technique used in the present study is not primarily designed to evaluate the magnitude of the neuroendocrine responses. Usually this is tested by injection of a standard dose of insulin [16, 17], or by clamping the blood glucose at a fixed, low level [18]. Since the insulin sensitivity and the hypoglycaemic threshold differs between individuals, these methods will give a hypoglycaemic stimulus that is very differently experienced. Accordingly, when comparing groups for neuroendocrine responses to hypoglycaemia with these methods, the hypoglycaemic thresholds should be similar if the comparison is to be valid.

In conclusion, we have found the present application of the glucose clamp technique to give reproducible results, revealing individual differences in glycaemic thresholds of neuroendocrine responses and symptoms to hypoglycaemia.

Acknowledgements. The present study was financially supported by the Norwegian Research Council for Science and the Humanities, Nordisk Insulinfond, the Norwegian Diabetes Association and the Norwegian Council on Cardiovascular Research. We wish to thank
Ms. T. Arnesen, Ms. R. Jæger, Mr. K. Mindeberg and Ms. T. Arild for their skillful technical assistance.

\section{References}

1. Amiel SA, Sherwin RS, Simonson DC, Tamborlane WV (1988) Effect of intensive insulin therapy on glycemic thresholds for counterregulatory hormone release. Diabetes 37:901-907

2. Mokan M, Mitrakou A, Raptis G et al. (1991) Differential effects of duration and glycemic control on thresholds and magnitudes of responses to hypoglycemia. Diabetes 40: 556A (Abstract)

3. Davis MR, Shamoon H (1991) Deficient counterregulatory hormone responses during hypoglycemia in a patient with insulinoma. J Clin Endocrinol Metab 72: 788-792

4. Snorgaard O, Lassen LH, Rosenfalck AM, Binder C (1991) Glycaemic thresholds for hypoglycaemic symptoms, impairment of cognitive function, and release of counterregulatory hormones in subjects with functional hypoglycemia. J Int Med 229: 343-350

5. Amiel SA, Simonson DC, Tamborlane WV, DeFronzo RA, Sherwin RS (1987) Rate of glucose fall does not affect counterregulatory hormone responses to hypoglycemia in normal and diabetic humans. Diabetes 36:518-522

6. Sager G, Trovik T, Slørdal L et al. (1988) Catecholamine binding and concentrations in acute phase plasma after surgery. Scand J Clin Lab Invest 48: 419-424

7. Hormonlaboratoriets analysebok (1991) Endokrine sykdommer og deres utredning, 9 utg. Aker sykehus, Oslo, p 172

8. Hormonlaboratoriets analysebok (1991) Endokrine sykdommer og deres utredning, 9 utg. Aker sykehus, Oslo, p 142

9. Jorde R, Burhol PG (1982) Effect of jejunoileal bypass operation and Billroth II resection on postprandial plasma pancreatic polypeptide release. Scand J Gastroenterol 17:613-617

10. Hepburn DA, Deary IJ, Frier BM, Patrick AW, Quinn JD, Fisher BM (1991) Symptoms of acute insulin-induced hypoglycemia in humans with and without IDDM. Diabetes Care 14:949-957

11. Widom B, Kinsley BT, Simonson DC (1991) Women and men differ in counterregulatory hormone responses to hypoglycemia. Diabetes 40: 543A (Abstract)

12. Diamond MP, Jones TW, Caprio S et al. (1991) Gender: determinant of the adrenergic response to hypoglycemia. Diabetes 40 : 27A (Abstract)

13. Mitrakou A, Ryan C, Veneman T et al. (1991) Hierarchy of glycemic thresholds for counterregulatory hormone secretion, symptoms, and cerebral dysfunction. Am J Physiol 260: E67-E74

14. DeFeo P, Gallai V, Mazzotta G et al. (1988) Modest decrements in plasma glucose counterregulation cause early impairment in cognitive function and later activation of glucose counterregulation in the absence of hypoglycemic symptoms in normal man. J Clin Invest 82: 436-444

15. Kerr D, Reza M, Smith N, Leatherdale BA (1991) Importance of insulin in subjective, cognitive, and hormonal responses to hypoglycemia in patients with IDDM. Diabetes 40: 1057-1062

16. Frier BM, Fisher BM, Gray CE, Beastall GH (1988) Counterregulatory hormonal responses to hypoglycaemia in type 1 (insulin-dependent) diabetes: evidence for diminished hypothalamic-pituitary hormonal secretion. Diabetologia 31: 421-429

17. Bernini GP, Argenio GF, Vivaldi MS et al. (1990) Impaired growth hormone response to insulin-induced hypoglycaemia in obese patients: restoration blocked by ritanserin after fenfluramine administration. Clin Endocrinol 32:453-459

18. Heller SR, Cryer PE (1991) Reduced neuroendocrine and symptomatic responses to subsequent hypoglycemia after 1 episode of hypoglycemia in nondiabetic humans. Diabetes 40: 223-226

Received: 20 February 1992

and in revised form: 29 May 1992

Dr. H. Vea

Department of Medicine

University Hospital of Troms $\emptyset$

N-9038 Tromsø

Norway 